\title{
ASPECTOS TERRITORIALES DEL SECTOR AGRARIO ANDALUZ
}

Manuel MARCHENA GOMEZ*

\section{INTRODUCCION}

Con este artículo se pretende, básicamente, aportar mapas actualizados de los espacios regionales cultivados siguiendo una metodología escasamente utilizada para el total de nuestra Comunidad Autónoma. Nos referimos al estudio con base municipal de las superficies de secano y regadío, que hasta ahora habían sido normalmente abordadas sobre delimitaciones comarcales y provinciales $^{1}$. Sin embargo, en segundo lugar, y luego de la valoración (tipología municipal) de los datos elaborados, plantearemos uno de los dilemas claves, desde nuestro punto de vista, en la ordenación territorial de estos recursos agrarios, cual es la necesaria articulación entre la actuación sectorial pública y la planificación espacial. Para ello, traemos a colación una problemática actualmente en curso de desarrollo: el tratamiento de la Agricultura en Areas de Montaña.

Es evidente que la política agraria en Andalucía, hoy, tiene como punto referencial la Ley de Reforma Agraria (OJEDA y OTROS, 1984). Sin embargo, es necesario abordar otros aspectos de un sector tan importante en nuestra región; que desde la perspectiva geográfica pasan ineludiblemente por la consideración territorial. En las siguientes líneas trataremos el significado espacial de la agricultura andaluza, pero insistiendo en la reflexión sobre el profundo contenido de ajuste o desarticulación en la organización del espacio (G. CANO, 1984), a la que pueden inducir las especificaciones territoriales de distintos cuerpos legales (en este caso la Ley y Reglamento de las Zonas de Agricultura de Montaña) referentes a la problemática agraria.

* Profesor del Departamento de Geografía. Universidad de Sevilla.

1. Henuos elegido exclusivamente los datos sobre superficies cultivadas, tanto en secano como en regadío soslayando para la cartografia las restantes variables de usos rurales (prados y montes) y no agrícolas, que concurren en el término municipal y que en otros apartados del artículo se utilizan. Los datos han sido elaborados en base a un listado (recabado de las Cámaras Agrarias Locales) del total de los municipios andaluces, donde se reflejan distintas variables de usos del espacio, gentilmente facilitado por la D.G. de Estructuras Agrarias de la Junta de Andalucía (1983), de donde posteriormente pudieran estudiarse otros aspectos. 


\section{EL MARCO TERRITORIAL DEL SECTOR AGRARIO ANDALUZ}

No es necesario destacar el trascendental peso específico que supone e sector agrario en nuestra región y en el factor determinante que éste asum en momentos de dilatadas crisis económicas (A.M. BERNAL, 1984, I y II dada la insuficiencia industrial o la coyunturaliedad que representa el turis mo, aún siendo este subsector, pieza clave en la economía regional: el campc andaluz ocupa el $22 \%$ de la población activa; genera el $13 \%$ del VA regio nal, aportando el 22,7 \% de la renta agraria de España (SANCHEZ, 1983). Y esta preeminencia de la función agraria en Andalucía (DRAIN, M. y ROUX B., 1978), también puede constatarse en su dimensión territorial (luego valo. raremos ciertas puntualizaciones sectoriales), basándonos en los datos de: cuadro I.

La superficia agraria útil (SAU), es decir las tierras de cultivo, prados y pastizales y los aprovechamientos forestales suponen el 86,2\% del territorio regional $^{2}$. Pese a ello, como lo han expresado numerosos autores, es necesario matizar el estereotipo de Andalucía como región llana y productiva (CRUZ VILLALON, 1983), ya que el área que ha marcado esa percepción del espacio andaluz solo supone un $1 / 5$ del total de éste, nos referimos naturalmente, al Valle del Guadalquivir. Mientras las otras dos grandes estructuras del solar regional, Sierra Morena y las Béticas (estas últimas constituyen $2 / 3$ del territorio), son áreas montañosas. Más tarde veremos la trascendencia del relieve en la caracterización de la superficie cultivada. Pero además, continuando con aspectos perceptivos, la expansión antes mencionada del regadío, que no cesa en la actualidad en las zonas litorales, mediante una agricultura forzada y especulativa, ha formalizado una nueva imagen de la agricultura andaluza (ZOIDO, 1982).

La provincia de Sevilla es la más favorecida tanto en superficie de secano $(19,7 \%$ de la región) como de regadío $(29,6 \%)$, mientras que el caso de Huelva es significativamente el inverso. Sin embargo, esta última circunscripción aporta porcentualmente cerca del $25 \%$ de la extensión forestal andaluza, aunque como todos sabemos cerca del $25 \%$ de aquella sea eucaliptal (PEPMF. Huelva, 1985). En cuanto a eriales y espartizales, aún cuando suponen sólo el 8,2 \% (cifra relativa, superior a la que aporta el regadío) de la superficie regional, representan en Almería el 35,5\%. Esta provincia junto con Granada suman más de la mitad de estas superficies no productivas o infraproductivas en Andalucía. En este sentido, siguiendo los criterios del Servicio

2. Según se recoge de A.M. BERNAL (1984), a mediados del Siglo XVIII, la mayor parte de las tierras andaluzas eran productivas $(68,4 \%$ ) y de ellas cultivables el $80 \%$. Hoy lo cultivable, por la asignación preferente del territorio a usos urbanos, se puede plantear más desde el angulo de reconversión de secanos en regadío o de la optimización de superficies forestales y no tanto como "frontera" de conquista hacia nuevos espacios cultivados. Ver J, OJEDA (1985). 
de Conservación de Suelos (USDA) sobre el $75 \%$ de los suelos almerienses soportan una erosión grave ${ }^{3}$, hecho contrastado con ser la zona andaluza con menores recursos forestales. La provincia con más posibilidades pratenses es Granada, aunque la aportación de su subsector ganadero solo sea del $20 \%$ de la producción final agraria, cerca de la media andaluza (19\%), pero muy lejana de la nacional que se encuentra sobre el $40 \%$, y de las aportaciones de Huelva $(29,6 \%$ ) y de Sevilla $(26 \%)$. Los terrenos propiamente improductivos varían territorialmente según el grado de impacto del suelo urbano (Sevilla, Málaga) o de las condiciones naturales (Almería, Granada). En Ríos y Lagos, destacan poderosamente las aportaciones de Huelva y Sevilla, Las Marismas, zonas húmedas y las cuencas del Guadalquivir, Tinto y Odiel, son la explicación (ver cuadro I).

Si repasamos los valores porcentuales respecto a los totales provinciales, se refleja como son las superficies de secano, al igual que en la región (40,7 \% ), las ocupaciones más importantes en los usos del suelo. Excepto en dos casos significativos y anteriormente aludidos, el de Huelva, cuyo terreno forestal supera con creces la mitad del territorio provincial $(65,1 \%)$ y el de Almería, ya que el erial y espartizal ocupan el 35,5\% de aquel. Se significa poderosamente el regadío de Sevilla, cuyas 175.880 ha. suponen el doble de la media regional $(6,5 \%)$ en valores relativos. El caso menos importante, dentro de su contexto superficial, sigue siendo el regadío de Huelva, no así en cuanto su dinamicidad y aportación económica (MARQUEZ DOMINGUEZ, 1984).

No queremos abundar en consideraciones expresadas de manera más directa en el cuadro I, solo indicar que las tierras no cultivadas en Andalucía suponen la mitad de su superficie, y que de ellas, el terreno forestal es el 30,3 $\%$. Aunque es necesario incidir en la idea de que este total de hectáreas (2.645.355) consideradas como forestales no necesariamente se encuentran arboladas. Asi el monte maderable supone el $41 \%$ de este terreno forestal, mientras que el monte abierto, es decir donde en teoría puede pastar el ganado se acerca al $36 \%$, superficie en general mayoritariamente no arbolada. El porcentaje restante se inscribe en el apartado del denominado "monte leñoso". El tema de la resforestación es clave para detener el peligroso proceso de desertización andaluz (el $40 \%$ de las tierras andaluzas están afectadas por una fuerte erosión); acción forestal que no solo podría aplicarse en áreas de montaña con distintas finalidades (ocio, madera, régimen hídrico, etc.), sino en terrenos campiñenses, especialmente bosques galerías, que permitirían una reconversión (aparte de otros beneficios tangibles), incluso paisajística del Valle.

3. Ver Catálogo de Suelos de Andalucía. Agencia de Medio Ambiente, Junta de Andalucia, Sevilla, 1984, pp. 271. Véase también MINISTERIO DE AGRICULTURA, ICONA. Paisajes erosivos en el Sureste español. Ensayo de Metodología para el estudio de su cualificación y cuantificación. Madrid, M. ${ }^{\circ}$ Agricultura, Pesca y Alimentación, 1982, 67 págs. (R.E.A. n. ${ }^{\circ}$ 3, pp. 193-195). 
CUADRO I

SUPERTICIES EN HA. DE USOS DE SUELO EN ANDALUCIA (1983)

\begin{tabular}{|c|c|c|c|c|c|c|c|c|c|c|c|c|c|c|c|c|c|c|c|c|c|}
\hline & \multicolumn{6}{|c|}{ Tierras de Culttvo } & \multirow{2}{*}{$\begin{array}{c}\text { Prados } \\
\text { y Pastizales }\end{array}$} & \multirow[b]{2}{*}{$1 *$} & \multirow[b]{2}{*}{$2=*$} & \multirow{2}{*}{$\begin{array}{l}\text { Terreno } \\
\text { Forestal }\end{array}$} & \multirow[b]{2}{*}{$1 *$} & \multirow[b]{2}{*}{$2 * *$} & \multirow{2}{*}{$\begin{array}{c}\text { Erial y } \\
\text { Espartizal }\end{array}$} & \multirow[b]{2}{*}{$1 *$} & \multirow[b]{2}{*}{$2 * *$} & \multirow{2}{*}{ Improductivo } & \multirow[b]{2}{*}{$1 *$} & \multirow[b]{2}{*}{$2 * *$} & \multirow{2}{*}{ Afos y Lagos } & \multirow[b]{2}{*}{ 1* } & \multirow[b]{2}{*}{$2 *=$} \\
\hline & Secano & $I^{*}$ & $2^{k *}$ & Regadio & $1 *$ & $2 * *$ & & & & & & & & & & & & & & & \\
\hline Almería ..... & 210.499 & 5,9 & 24 & 65.746 & 11.1 & 7,6 & 73.209 & 9,8 & 8,4 & 147.104 & 5,6 & 16.8 & 311.631 & 43,3 & 35,5 & 58.855 & 15,6 & 6,7 & 8.856 & 7,8 & 1 \\
\hline Cadiz . . . . . . & 300.865 & 8,5 & 40,7 & 34.794 & 5,8 & 4,7 & 101.908 & 13.7 & 13 & 229.541 & 8,7 & 31,2 & 18.122 & 2,5 & 2,5 & 39.196 & 10,3 & 5,3 & 14.285 & 12,7 & 1,9 \\
\hline Córdoba ...... & 661.879 & 18,7 & 48,1 & 66.204 & 11,1 & 4,8 & 105.159 & 14,1 & 7,1 & 469.516 & 17,7 & 34,2 & 18.700 & 2.6 & 1.3 & 37.241 & 9,8 & 2,8 & 15.997 & 14,2 & 3,1 \\
\hline Granada , . . . & 523.679 & 14,8 & 41,7 & 106.285 & 17,9 & 8,4 & 174.801 & 23,5 & 13,9 & 264.856 & 20,1 & 21,5 & 125.914 & 17.5 & 10,1 & 55.303 & 14,6 & 4,4 & 5.466 & 4,8 & 0,4 \\
\hline Huelva ....... & 187.289 & 5,3 & 18,6 & 9.588 & 1,9 & 0,9 & 30.354 & 4,1 & 3,1 & 653.698 & 21.1 & 65,1 & 66.544 & 9,3 & 6,6 & 30.367 & 8,1 & 3.1 & 26.364 & 23,4 & 2,6 \\
\hline Jaén. . . . . . . & 637.175 & 17,9 & 46,7 & 85.427 & 11,5 & 6,3 & 128.551 & 17,3 & 4,5 & 409.855 & 15,5 & 30,2 & 40.531 & 5.6 & 2,9 & 50.724 & 13,4 & 3,7 & 9.981 & 8.7 & 0.7 \\
\hline Málaga . . . . . & 324.391 & 9,2 & 44,9 & 48.818 & 8,1 & 6,8 & 2.626 & 0,4 & 0,4 & 190.685 & 7,2 & 26,4 & 103.746 & 14,4 & 14,4 & 41.968 & 11,1 & 5.8 & 9.692 & 8.6 & 1.3 \\
\hline Sevilla $\ldots \ldots$ & 696.327 & 19,7 & 49.2 & 175.888 & 29.5 & 12,5 & 126.927 & 17,1 & 9,0 & 280.100 & 10,5 & 20,2 & 33.291 & 4,7 & 2,5 & 65.133 & 17,1 & 4.9 & 22.394 & 19,8 & 1,7 \\
\hline ANDALUCIA . . & 3.542 .104 & 100 & 40,7 & 592.742 & 100 & 8,7 & 743.535 & 100 & 8,7 & 2.645 .355 & 100 & 30,3 & 718.479 & 100 & 8,2 & 378.687 & 100 & 4,3 & 113.035 & 100 & 1,3 \\
\hline
\end{tabular}

ELABORACION PROPIA a partir de los datos municipales de las Cimaras Agrarias.

1** Porcentaje respecto al total regional, 


\section{LA DISTRIBUCION DE LA SUPERFICIE CULTIVADA EN ANDALUCIA}

La metodología utilizada para la confección del primer mapa, es la de cartografiar por términos municipales la superficie cultivada, tanto secano como regadío, con respecto al total territorial del municipio. Relación que por su sentido intrínsecamente espacial, es decir de extensión en el suelo, genera una fuerte expresividad en la base municipal; nuestro intento se dirige a una percepción rigurosa y totalizadora del territorio agrícola andaluz. En busca de esta visualización, hemos segmentado los valores en cinco grandes intervalos sobre el $100 \%$ de superficie cultivada. Además, se ha precisado en el mapa, los municipios afectados por la primera delimitación perimetral de las superficies susceptibles de ser declaradas, Zonas de Agricultura de Montaña (Orden 10540/1985), para su posterior comentario (ver mapa).

Andalucía representa el 17,3\% del Estado español, sin embargo aporta en distintos rendimientos agrícolas y en porcentajes relativos (regadío, por ejemplo) valores superiores a las medias nacionales (GONZALEZ DELGADO, 1979). Esta visión genérica de nuestra región como área privilegiada en recursos naturales, y más concretamente en potencialidades agrícolas no es generalizable a la vista del mapa, o de los datos del punto anterior. No todo es espacio cultivado ni buena agricultura. Es necesario plantear la diversidad del área cultivada andaluza ("Las agriculturas andaluzas"), en distintos espacios agrarios que permitan una mejor comprensión de la realidad global: campiña, regadíos, altiplanicies, montaña, agricultura litoral etc., a la vez que precisar que cultivos tradicionales de la trilogía mediterránea (tan ensalzados por los viajeros románticos), componen áreas de fuerte homogeneidad; el olivar jiennense; el viñedo jerezano, pueden ser ejemplo de lo que decimos. En último término tal como lo propone el mapa, el paisaje cultivado dibuja un conjunto de regiones homogéneas bastante ligadas a las condiciones del medio físico (CANO, 1983).

Efectivamente, por una parte, la expresividad y el diseño preciso del Valle a través de las superficies cultivadas que suponen más del $80 \%$ de ocupación del suelo de la Depresión Bética. Por otra, la escasa superficie cultivada de Sierra Morena (y en general de la provincia de Huelva) cuyos municipios en contadas ocasiones llegan al $40 \%$ de la superficie cultivada ${ }^{4}$. Y al Sur, un grado de mayor complejidad, pero muy relacionable con la estructuración de

4. Es necesario destacar, ya que asi resulta en el mapa, el caso de la comarca de Los Pedroches cuyo subsuelo es un extenso batolito granítico, que topográficamente se manifiesta en un relieve plano; produciéndose una mayor capacidad agronómica que en el resto de Sierra Morena. De ahí que la intensidad de cultivos (olivar, sobre todo) destaque poderosamente en su contexto subregional. 


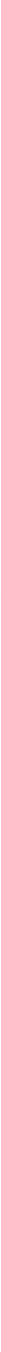


las Béticas: los "vacíos" en el mapa correspondientes a Sierra Nevada y la Serranía de Ronda, y las Vegas intermedias, cultivadas intensamente, de Guadix, Granada y Antequera, correspondientes al Surco Intrabético, por ejemplo, nos muestran la fuerte correlación que aparece en Andalucía entre cultivo y grandes estructuras físicas, cuya articulación, como decimos, se dibuja expresivamente en el mapa.

Haciendo un repaso global por intervalos, podemos precisar que las áreas con menos del $20 \%$ de superficie cultivada son: en general, la mitad norte de la provincia de Huelva (Andévalo y Sierra), con alguna excepción en la comarca de Aracena; aparte de municipios como Encinasola (al igual que ocurre con Guadalcanal en Sevilla y en general en Los Pedroches) que sobrepasan el dibujo de la alineación de Sierra Morena, participando así, más de los rasgos meseteños. Espacios mínimamente cultivados, siguiendo el borde norte del Valle, continúan siendo las provincias de Sevilla (Constantina supera el $20 \%$ por la presencia fuertemente olivarera), Córdoba y Jaén. En esta última además destaca poderosamente, por debajo del $10 \%$ de la superficie cultivada, los municipios enclavados en las formaciones más potentes de la Sierra de Segura (una media de 1.500 metros de altitud). Excluida Sierra Morena $\left(18.000 \mathrm{~km}^{2}\right)$ se perciben otras cuatro áreas en este intervalo inferior. La primera, la zona marismeña centrada en Almonte; luego, siguiendo hacia el Este, los enclaves montañosos de la Serranía de Ronda y el Aljibe y el núcleo de Sierra Nevada y la Sierra de Gádor, y por fin, un área peor dibujada en el centro de la provincia de Almería, entre las estribaciones de la Sierra de Filabres y el Campo de Tabernas.

Entre el 20 y el $40 \%$ de superficie cultivada, se encuentran espacios de transición entre las comarcas anteriormente reseñadas y zonas más intensivamente cultivadas (Huelva o la fachada suratlántica gaditana). Destacan además puntos más homogéneos hasta el $40 \%$ de superficie cultivada, la Comarca del Alhama y el Valle de Lecrín, ambas en Granada. A caballo con el siguiente intervalo, es decir hasta el $60 \%$ se encuentran genéricamente las Altiplanicies Orientales (acentuándose el porcentaje en la parte sur de los Vélez); que suman 200.000 ha. de alto monocultivo cerealísta, ocupando el 90 $\%$ de lo cultivado en el nordeste andaluz. En situación de porcentajes similares se encuentra la mencionada comarca olivarera de Los Pedroches y de Montoro. Mientras que áreas definidas claramente por el intervalo intermedio (40-59\%), solo encontramos, la costa malagueña, especializada en el sector turístico, más una parte del sur jiennense (La Mágina); además de los casos de menor homogeneidad (Altiplanicies) mencionados anteriormente.

Las realidades más uniformes se conforman en los intervalos superiores; sobre el $70 \%$ de superficie cultivada se sitúan las comarcas de Antequera, el marco de Jerez y toda la zona de especialización olivarera del subbético cordobés (ORTEGA ALBA, 1974), con sus prolongaciones en las provincias de 
Jaén y Granada (Los Montes), por la perfecta adaptación de este tradici leñoso mediterráneo a la topografía y los terrenos calizos. La importanci este cultivo en Andalucía (incluso desde el punto de vista de la identifica perceptiva de la región) nos obliga a decir, siguiendo a LOPEZ ONTIVER Ag. (1982), que la falta de competitividad de muchas áreas marginales de var con los cultivos herbáceos oleaginosos y la entrada en la CEE, tend a hacer desaparecer por deseconomía, a los olivares que produzcan meno $1.000 \mathrm{~kg}$./ha. que suponen, por ejemplo, el $31 \%$ del olivar de Jaén y e $\%$ del malagueño.

Descontando estas anteriores zonas, aparecen con un índice de alta perficie cultivada, en Granada, la Vega en torno a la capital, cuyo paj agrario surgió a causa del regadío pero que prácticamente no ha salido de límites que tuviera en la época musulmana, ocupando el $50 \%$ de la sup cie cultivada (OCAÑNA, 1974) y el área litoral, especializada en cultivos ex cos (chirimoya, aguacate). Pero, como decíamos al principio de este ape do, quizás lo más destacable visualmente del mapa es toda la superficie । tral, correspondiente al Valle del Guadalquivir, Vega y Campiña, que so pasa con creces el $80 \%$ de superficie cultivada sobre el total del territoric

Mucho se ha hablado, pese al alto porcentaje de tierras agrícolas del lle, de su infrautilización económica e injusta distribución de la propieda del dispendio del regadío (ZOIDO, 1980); se aprecia, por otra parte, un cesivo monocultivo del olivar en algunas áreas (Jaén cuenta con 28.650 ha La escasa importancia de frutales y cultivos forrajeros y el aprovechamie antisocial en zonas de regadío, de cereal, cuyos rendimientos superan el ble de la media nacional, generando una renta diferencial de más del 50 (asegurada hasta ahora por el proteccionismo oficial), son entre otras, las funcionalidades señaladas por ERA (1980). La reforma agraria que ah se comienza a desarrollar, y no es casualidad, en sus cuatro primeros De tos y Actuación Comarcal designa dos áreas de máximo aprovechamientc superficie cultivada y regada: las Vegas de Sevilla y de Córdoba. En gene y según datos del propio IARA, en base al Censo Agrario de 1982, la forma Agraria va a actuar en Andalucía en 4.807 explotaciones de más 300 ha. compensadas $^{6}$ y que suponen algo así como el $51 \%$ de las tierras secano y el $38 \%$ de la superficie de regadío.

5. Al estrangularse la Depresión en dicha provincia entre las formaciones de Sierra Morena y las 1 cas Internas, se constituye un inmenso olivar confluyente en estas unidades, hasta que en el. Guadalquivir se dispersan progresivamente por la altitud y topografía más adversa (Sierr: Segura).

6. Es decir, evitando tecnicismos estadísticos, aquellas que no están exentas del impuesto de ini tilización: fincas mayores de 50 ha. en cultivo de regadío, de más de 300 ha. de cultivo de sec y superiores a 750 ha. de pastos o monte (Ley de Reforma Agraria, art. 36, punto 5). 


\section{DISTRIBUCION ESPACIAL DE LAS SUPERFICIES REGADAS}

El segundo mapa elaborado intenta matizar (siguiendo la línea del anterior en cuanto a expresividad) las superficies cultivadas, mediante la representación del regadío según los porcentajes municipales de tierra regada con respecto al total del suelo cultivado; ya que las cifras relativas sobre el total territorial reflejarían valores de escasa importancia numérica y complicarían la interpretación cartográfica. Para evitar también el sesgo que pudiera producirse al representar los parámetros del regadío en pequeños municipios (que distorsionan el contexto general al ser porcentajes representativos de pocas héctareas) hemos mapificado con trama rayada, los municipios con más de 1.000 ha. de regadío que no alcanzan el $20 \%$ con respecto al total cultivado, y que se corresponden generalmente con entidades locales de extensión considerable ${ }^{7}$.

El regadío andaluz cuya antigüedad puede remontarse a la época romana, pero su desarrollo más efectivo se realiza bajo el dominio islámico, hasta principios de siglo, por las aludidas causas históricas, marcaba diferencias entre el área oriental y occidental de la región. En la primera, las Vegas granadinas del Genil (Granada, Loja) y Guadiana Menor (Guadix, Baza, Huéscar) son el paradigma del regadío secular andaluz (BOSQUE MAUREL, 1980). Sin embargo es desde inicios de nuestro siglo, sobre todo desde los cincuenta y a partir de las actuaciones del IRYDA (ROMERO, J.J. y ZOIDO, F., 1977), cuando el regadío de la Depresión Bética, es cuantitativamente el de mayor peso específico en Andalucía (ver cuadro I), suponienco una de las zonas regadas más importantes en el conjunto nacional y un enclave de dinamización y atracción demográfica en la región (JORDA BORRELL, 1985).

De esta manera se dibujan en el mapa tres significativas superficies de regadío: las grandes zonas regables de las cuencas del Guadalquivir, Guadalete y Barbate, a cuenta del erario público ${ }^{8}$; los regadíos tradicionales del oriente andaluz $\mathrm{y}$, por último, la agricultura por enarenados y forzada del litoral, que pese a lo reducido de su extensión genera el $20 \%$ de la producción final agraria en Andalucía (ERA, 1980). Se denotan además algunos enclaves residuales, significativos de antiguas economías tradicionales cerradas, como es el caso de Galarosa en Huelva (SANTOS, N. y ZOIDO, F., 1980), hoy en proceso de desarticulación. Otro hecho expresivo, se deduce de la comparación del mapa de superficies cultivadas y el de porcentajes de regadío. Nos

7. Esta decisión se ha tomado al comprobar que municipios que no llegan a este $20 \%$, tienen más hectáreas regadas que el total de la provincia de Huelva, por ejemplo. Caso de Carmona o de Jerez de la Frontera.

8. Actuaciones oficiales fundamentalmente iniciadas, como decíamos en los años cincuenta; en total 24 zonas regables, que han beneficiado a 85.000 ha. campiñenses. El cultivo del arrozal marismeño se inició (hoy, $40 \%$ de la superficie nacional de esta gramínea), con anterioridad, en los años treinta. 


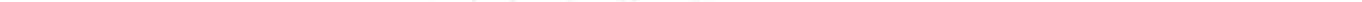


referimos a la complementareidad existente en ciertos espacios de Granada y Almería (sobre todo Las Alpujarras), entre la ausencia de superficie cultivada apreciable, que solo se manifiesta a través del regadío (de ahí los elevados porcentajes de éstos). Es decir las condiciones medioambientales posibilitan exclusivamente en estas zonas una agricultura basada en la seguridad e intensidad del regadío; en los suelos donde es posible plantar (fondos de valle, bancales).

Los porcentajes más importantes de aprovechamientos en regadíos se dan principalmente en el sector oriental anteriormente mencionado (incluyendo municipios de la Vega de Granada, Lecrín, Alpujarras y valles del Andarax y Almanzora) (HERNANDEZ, J., y MARTINEZ, F., 1980) y en la Vega del Guadalquivir (Brenes y Tocina en Sevilla cuentan con una superficie cultivada $100 \%$ de regadío) y las Marismas. Esto si nos referimos a los municipios con regadío superficial, ya que el Campo de Dalías, funciona sobre acuíferos (al igual que buena parte del regadío de Almonte). Los porcentajes relativos al regadío tambien aqui son muy altos, en un sistema de cultivos bajo plásticos, desarrollado desde 1956 y que actualmente ocupa 12.000 ha., necesitándose en la actividad de agricultura forzada desarrollada, una enorme carga de inputs (luego amortizados, sobre 1.300 .000 ptas./ha. GARCIA MANRIQUE, 1984).

La política de futuro en el regadío andaluz, que debe por otra parte ajustar debidamente el discurso ideológico de panacea que algunos autores le han designado, con el de mejor distribución pública de las inversiones y de la propiedad; pasa por una consumición óptima de los recursos regulados ya que los volúmenes hoy consumidos son muy elevados, distanciándose bastante de las cifras de consumo teórico ideal (VARIOS, 1979). Aparte de la debida atención que merece la vulnerabilidad y uso de acuíferos, aspectos tratados en los Planes Especiales de Protección del Medio Físico provinciales y PDTC de Doñana, y que en el caso de Almería, plantea una problemática difícil por la sobreexplotación del agua subterránea.

\section{MODELOS DE DIVERSIDAD AGRARIA EN ANDALUCIA}

La revisión de trabajos comarcales y sobre geografía rural en nuestra región, junto con la síntesis de datos municipales expresados en los mapas anteriores, nos han hecho reflexionar sobre la necesidad de confeccionar un cuadro-resumen. En éste, las grandes áreas con aprovechamiento desigual de su superficie cultivada y recursos agrarios, en nuestra Comunidad Autónoma, 
han sido representados por municipios tipológicos, según la utilización que hacen o pueden hacer de su suelo. Se intenta, como objetivo, elaborar un pequeño compendio con porcentajes modélicos de los elementos integrantes de la Superficie Agraria Util (SAU), en los distintos espacios subregionales caracterizados; éstos se ejemplificarán en uno o varios municipios que contengan las coordenadas medias de su área o subáreas (al comprobarse espacios discriminados en las subregiones. Ver cuadro II).

Se ha relacionado la SAU con el total de término municipal elegido (productivo e improductivo), porcentuándose luego, la superficie cultivada, prados y pastizales y terreno forestal, con la SAU municipal respectivamente. Evidentemente, se trata de un proceso de simplificación de la realidad con el único objetivo de reunir a "golpe de vista" y de manera cuantitativa, las diversidades agrarias andaluzas. Esta tipología puede completarse con la obtenida en base a nueve indicadores de índole económico en PAYNO y OTROS (1983, pag. 168-169), tres de ellos relativos a productividad de los factores (del empleo, del suelo, empleo/superficie), tres a inversión (por superficie, por empleo y propensión a ella) y otros tres, sobre la forma de explotación (superficie media, asalariados y mecanización). Sin embargo ésta, está diseñada a nivel provincial, aunque en las conclusiones que se obtienen, los autores marcan los diferentes espacios subregionales en función de cuatro áreas. Por una parte, las tres provinciales que componen el llamado "Eje Interior" (Córdoba, Jaén, Granada), donde todos los indicadores se mantienen en niveles bajos. En segundo lugar, la denominada "Andalucía del Estrecho", se segmenta, ya que Cádiz y Sevilla aportan unos valores de tono medio, con una intensidad de empleo comparativamente baja; mientras que Málaga se caracteriza por una fuerte capitalización por superficie, una intensidad de empleo y una productividad relativamente bajas, que se corresponden con un tamaño de explotaciones muy bajo. La "Andalucía Levantina" (Almería), marcada por la potencia de la capitalización y del empleo, y en consecuencia, por la intensidad de la productividad de la tierra. Huelva (La "Andalucía Atlántica") finalmente, presenta un perfil asimétrico, puesto que es una agricultura extensiva de poca productividad y empleo, en la que sin embargo, la capitalización juega un papel principal respecto a otros factores productivos.

Estas consideraciones (ver MARTINEZ SIERRA, 1982), como decimos, tienen una visión sensiblemente más económica y se basan en argumentos a nivel provincial. El cuadro II, intenta sobrepasar las barreras administrativas y adentrarse en un intento tipológico de alcance territorial (usando indicadores contrastados) y en orden a la extensión de este artículo. Pensamos que el cuadro evita comentarios en exceso, ya que su finalidad es destacar las diferencias espaciales y sintetizar argumentos expuestos anteriormente, como complemento a la cartografía realizada. Sin embargo se podrían realizar dos puntualizaciones: 


\section{CUADRO II}

TIPOLOGIA AGRARIA EN ANDALUCIA (DATOS EN \%). BASE MUNICIPAL

\begin{tabular}{|c|c|c|c|c|c|}
\hline Subregión & Municipio-Tipo & SAU/Total Municipal & S./Cultivo. SAU (*) & Prados/SAU & Forestal/SAU \\
\hline \multirow[t]{6}{*}{ Sierra Morena } & Huelva: & & & & \\
\hline & - Aracena . ...... & 98,8 & $20,4(1,3)$ & 1,3 & 78,3 \\
\hline & Cordoba: & & & & \\
\hline & - Hornachuelos. . . . . . & 95,5 & $18,7(54,7)$ & 16,4 & 64,9 \\
\hline & Los Pedroches: & & & & \\
\hline & - Hinojosa del Duque. . & 94,0 & $70,2(0,8)$ & 3,1 & 26,7 \\
\hline \multirow[t]{2}{*}{ Campiñas del Guadalquivir } & 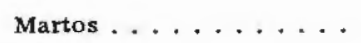 & 95,6 & $96,8(3,3)$ & 1,5 & 1,7 \\
\hline & 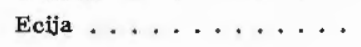 & 94,7 & $99,0(15,8)$ & 0,7 & 0.3 \\
\hline \multirow[t]{4}{*}{ Vega del Guadalquivir } & Ribera/Campiña: & & & & \\
\hline & - Palma del Río ..... & 96,4 & $96,6(51,1)$ & 3,1 & 0,3 \\
\hline & Ribera: & & & & \\
\hline & - Brenes......... & 82,2 & $100 \quad(100)$ & 0 & 0 \\
\hline
\end{tabular}

Nuevos Regadios

de la Depresion Bética

Area de Jaén:

- Mancha Real ......

98,9

$92,5(28,7)$

0,3

7,1

Las Marismas:

- Los Palacios. ......

95,9

$98,5(76,2)$

1,5

0

Guadalcacín:

- Jerez de la Frontera ..

93,8

$73,8(13,7)$

10,4

15,8 
CUADRO II (Continuación)

\begin{tabular}{|c|c|c|c|c|c|}
\hline \multirow[t]{8}{*}{ Subbético } & Alto Guadalquivir: & & & & \\
\hline & - Cazorla ........ & 98,0 & $38,4(8,8)$ & 19,1 & 42,4 \\
\hline & Los Montes: & & & & \\
\hline & - Priego de Córdoba. . . & 70,3 & $82,8(3,7)$ & 3,1 & 14,1 \\
\hline & Antequera: & & & & \\
\hline & - Antequera. . . . . . & 83,9 & $79,3(15,7)$ & 0,2 & 20,5 \\
\hline & Serranía de Ronda: & & & & \\
\hline & - Ronda.......... & 79,6 & $58,3(3,3)$ & 0,2 & 41,5 \\
\hline \multirow[t]{2}{*}{ Altiplanicies Orientales } & Vélez Blanco. . . . . . . . & 31,2 & $45,3(3,7)$ & 3,9 & 50,8 \\
\hline & Baza $\ldots \ldots \ldots$ & 92,7 & $23,6(44,4)$ & 15,8 & 60,5 \\
\hline \multirow[t]{4}{*}{ Regadío Trađicional Oriental } & Vega: & & & & \\
\hline & - Churriana de la Vega. . & 54,3 & $100(100)$ & 0 & 0 \\
\hline & Alpujarras: & & & & \\
\hline & - Capileira. . . . . . . & 94,7 & $16,5(100)$ & 63,6 & 19,9 \\
\hline \multirow[t]{5}{*}{ Regadio Litoral Meditérraneo } & Area Monoturistica: & & & & \\
\hline & 一 Estepona . . . . . . & 80,5 & $36,7(40,2)$ & 8,9 & 54,4 \\
\hline & Agricultura Subtropical y & & & & \\
\hline & Enarenados: & & & & \\
\hline & -Almuñécar. . . . . . & 36,3 & $98,2(62,8)$ & 0,2 & 1,6 \\
\hline \multirow[t]{4}{*}{ Regadío Litoral Atlántico } & Cádiz: & & & & \\
\hline & - Chipiona $\ldots \ldots$ & 92,4 & $95,6(30,9)$ & 0,1 & 4.3 \\
\hline & Huclva: & & & & \\
\hline & 一 Isla Cristina. . . . . . & 83,5 & $53,5(53,1)$ & 0,3 & 46,3 \\
\hline
\end{tabular}


a) En todos los casos, la SAU supera el $80 \%$ sobre el total municipal excepto, en sectores de las Béticas Internas (Los Montes); de los regadíos tradicionales orientales (escasa aptitud del medio físico en el conjunto del término municipal: este se "reparte" alicuotamente entre diversos usos, para no primar excesivamente a una entidad); en zonas de las Altiplanicies Orientales almerienses, sobre todo Vélez Blanco y tramos de agricultura litoral mediterránea: la montaña solo permite una banda litoral, además de valles fluviales, con posibilidades de adaptación agraria.

b) También lo más frecuente es que la SAU se dedique esencialmente a agricultura. Las zonas forestales destacan lógicamente en Sierra Morena (con la excepción mencionada de Los Pedroches), en Cazorla y en áreas de las Altiplanicies Orientales, mientras que en otras es similar al aprovechamiento agrícola y forestal. Cosa que ocurre igualmente en la Serranía de Ronda y en algunos puntos del regadío litoral Atlántico. Solo el pastizal es predominante en el municipio representativo de Las Alpujarras, Capileira.

\section{SECTOR Y ESPACIO AGRARIOS}

Una de las carencias básicas en la planificación española es la necesaria articulación entre las medidas sectoriales y su coordinación a nivel territorial (recordar en este sentido, el fracaso y el difícil manejo de los PDTC. Ley del Suelo, 1975). Hasta ahora hemos comprobado las potencialidades agrícolas, y el reparto de éstas, por el solar andaluz. A simple vista son muchas, pero infrautilizadas. Nos encontramos en un período donde desde distintos ámbitos se aboga por la intervención reguladora de las instancias oficiales, al menos en un sentido directriz ${ }^{9}$, sobre todo de cara a la integración en la CEE (encuadrarse en la reestructura PAC, Política Agraría Común). Parece que hoy más que nunca se necesite un esfuerzo encaminado a que las diversas estrategias estatales y autonómicas, confluyan directamente en ámbitos territoriales precisos a reconvertir o a potenciar. Así organismos como el IARA, contemplan como base de operaciones esenciales, las actuaciones comarcales escalonadas, en un intento de no desenvolverse exclusivamente en macrolineas; pero a la par interpretando que es necesaria la convergencia en la misma institución, de las variadas competencias, que desde diversos organismos y regulaciones legales, impacten sobre la configuración del espacio agrario. Es un tema que incluso se pretende, con las obvias diferencias, en algunas experiencias municipales, caso de Lebrija (Plan de Desarrollo Comunitario). Es decir,

9. El término "Directriz" entendido como recomendación general de un organismo a otras instancias para que desarrollen sus instrumentos y leyes para su aplicación concreta. 
se trata de asignar territorialmente (una de las razones del diseño de Ambitos Funcionales por la Junta de Andalucía) las distintas inversiones y gestionar covenientemente éstas, según las prioridades.

Si en las páginas anteriores hemos analizado el espacio agrario, esencialmente la superficie cultivada, tratemos ahora de sintetizar cuales son las condiciones económicas del sector; los esfuerzos de la Comunidad Autónoma en proyectarlo y el comienzo del "futuro europeo", con la petición de fondos FEDER (Fondo Europeo de Desarrollo Regional):

1. Para radiografiar la problemática del sector agrario andaluz, seguiremos el estudio Claves para el Desarrollo económico de Andalucia (PAYNO y OTROS, 1983). En él se especifica que pese a indicadores que revelan una posición favorable del agro regional en el conjunto nacional (VAB, por ejemplo, o regadíos), el papel predominante de la agricultura en el sector agrario, no es suficientemente dinamizador ni su estructura conveniente. Ocupa un lugar trascendental en Andalucía, especialmente, por la ausencia de otros sectores más productivos, con un rendimiento general no sustancialmente alto. La productividad de la SAU está por encima de la media española, pero con una contrastada insuficiencia en producción ganadera y de huerta que expresa la escasa especialización del sector. Ello provoca, con otros factores, el exceso de mano de obra subempleada en la agricultura, sin alternativas en otros sectores para su absorción. De esta manera, las medidas aconsejadas fundamentalmente son, la pronta especialización de cultivos y productos, racionalizando las explotaciones y aumentando las extensiones de regadío. Estos objetivos básicos deben ser el reforzamiento de las interrelaciones técnicas entre agricultura e industria; fomentándose el cooperativismo y tejiéndose una malla integradora de todas las fases productoras hasta su comercialización. Se concreta que las acciones, en el contexto espacial, deben comenzarse prioritariamente en todos los ordenes, en el "Eje Interior" (Córdoba, Jaén, Granada), que según los autores del estudio, es absolutamente estratégico para retener población (ver MARCHENA GOMEZ, 1984, pag. 132), para difundir espacialmente los efectos que se obtengan y para reequilibrar las rentas intraregionales. Es decir, se recomienda expresamente el área a donde debe concretarse territorialmente, los esfuerzos en la tarea de coordinar las acciones correctoras para reconducir el sector agrario y en general, la economía andaluza.

2 El peso fundamental del sector agrario se sigue proyectando en las inversiones públicas a realizar por el Plan Económico de Andalucía 1984-86. Donde los objetivos sectoriales del Programa Agrario (basado literalmente en un "pleno aprovechamiento de los recursos productivos propios") alcanzan el 24,8\% de las asignaciones de la Administración Central y 
Autonómica de 1984. Ello supone un aumento del 71,9 \% de la tendencia de inversión pública del período 1978-82; constituyéndose de esta manera uno de los objetivos básicos del mencionado Plan, ya que absorbe el $54,2 \%$ de los recursos autónomos de inversión. Pero quizás, sea más contundente el dato, sobre las actuaciones del Programa Agrario de la Comunidad Autónoma, que tienen un efecto territorial aislable sobre la renta o el nivel de ocupación y que representan el 73,2\% del conjunto de inversiones previstas para 1984 en este sector. De los cuales 21.000 millones de ptas., corresponden al IARA ${ }^{10}$. De estos porcentajes rápidamente se deduce la concentración de esfuerzos públicos basada, según se lee en el Programa Agrario de la Comunidad Autónoma, en la creación de Infraestructura productiva y social y en la incentivación de la actividad privada mediante la subvención, y que por supuesto deberá tener un importante (positivo o negativo) reflejo en la ordenación general del territorio andaluz. Físicamente, ya sea por la creación de regadíos, expropiaciones, repoblación forestal etc., o indirectamente por la canalización de dinero público a determinadas áreas de población, por ejemplo ${ }^{11}$.

3. Las ayudas solicitadas en el ámbito de Andalucía para obtener financiación procedente del Fondo Europeo de Desarrollo Regional (FEDER) totalizan la cifra de 17.327 millones de ptas. (ver cuadro III). Los sectores que más volumen de financiación han generado son agricultura e infraestructura. Destaca el importante conjunto de recursos que demanda a trámite ante la CEE, el IARA: más de 5.000 millones de pesetas (5.112.184.171). Estos fondos suponen el $30 \%$ exclusivamente, de los más de quince mil millones como cifra de inversiones, que prevee el IARA en estas partidas. Los objetivos de estos programas tienen unas consideraciones muy importantes de cara a la organización del espacio: compra de fincas, reforestación, ordenación de cultivos, construcción y mejora de caminos rurales, electrificación y obras de encauzamiento de ríos. Los fondos destinados específicamente al sector agrícola se invertirán en la creación de empresas agroalimentarias y en el fomento de cultivos acuícolas.

Estos propósitos de reestructuración del sector agrario andaluz pueden entrar en conflicto, al no contemplarse con tino lo que supone explícitamente la acción territorial, es decir, la delimitación espacial y las circunstancias

10. Véase Criterio de Asignación Territorial de Inversiones de las Directrices Regionales y Experiencia comparada. D.G. Ordenación del Territorio, Consejería de Política Territorial, Junta de Andalucia.

11. En este sentido el representante de FASAGA, en una mesa redonda celebrada en la Facultad de Geografía e Historia de Sevilla el 18-1-85 (Ver R.E.A. n. ${ }^{\circ} 4$, p. 229), se quejaba ante el Viceconsejero de Agricultura, Antonio Gamir, de que las inversiones directas destinadas a Reforma Agraria (sobre 14.500 millones de ptas.) podrian drenarse más efectivamente para la economía regional, hacia otros sectores más productivos, según su opinión, como el turismo o la construcción. 
que concurren en cada ámbito. La planificación y los instrumentos sectoria. les, tarde o temprano, se cristalizan univocamente en la ordenación del terri. torio. El marco general económico debe procurar, en sus perspectivas de de. sarrollo, el ajuste entre territorio y sus recursos y la población que sustenta. En esta línea hemos intentado analizar en el próximo apartado, una medida concreta que intenta resolver estos asuntos y que afecta a zonas especialmen. te perfiladas por tensiones de diversa índole (ORTEGA VARCARCEL, 1985): la Ley de Agricultura de Montaña y por supuesto en lo que respecta a Andalucía.

CUADRO III

DISTRIBUCION SECTORIAL DE LOS FONDOS SOLICITADOS AL FEDER (1985-86)

\begin{tabular}{|c|c|c|c|}
\hline Areas & $\begin{array}{l}\text { Gastos sobre los que } \\
\text { se solicita ayuda }\end{array}$ & $\begin{array}{l}\text { Montante de ayudas } \\
\text { pedidas al FEDER }\end{array}$ & Empleos \\
\hline$\ldots \ldots \ldots$ & 807.670 .004 & 286.561 .316 & 480 \\
\hline Turismo $\ldots \ldots \ldots \ldots$ & 16.000 .000 .000 & 3.649 .100 .000 & 2.200 \\
\hline Infraestructura $\ldots \ldots \ldots$ & 33.845 .649 .000 & 12.136 .154 .777 & 11.761 \\
\hline Medio Ambiente $\ldots \ldots \ldots$ & 7.342 .893 .320 & 2.475 .999 .873 & 8.012 \\
\hline Transportes ......... & 1.917 .224 .144 & 680.231 .126 & 1.633 \\
\hline Agricultura. . . . . . . & 20.915 .911 .171 & 5.268 .750 .828 & 11.509 \\
\hline
\end{tabular}

Fuente: Consejería de Economía, Plamificación, Industria y Energía.

\section{UN INTENTO DE INTEGRACION SECTORIAL Y TERRITORIAL: LA LEY DE AGRICULTURA DE MONTA NA}

En los últimos meses, y luego de la Ley de Reforma Agraria quizá haya sido el desarrollo de la Ley de Agricultura de Montaña (1982) el punto donde se hayan centrado expectativas entre los técnicos ${ }^{12}$ de cambios concretos en territorios marcados por la primacía agraria y, en este caso, por ciertos rasgos físicos propios y de fuerte cariz geográfico ${ }^{13}$.

12. En este sentido se enmarca el Congreso sobre Agricultura y Desarrollo Rural en Zonas de Montaña. Celebrado en Granada del 4-8 de Noviembre de 1985 y organzado por la Consejería de Agricultura y Pesca de la Junta de Andalucía.

13. La Ley de Agricultura de Montaña (25/1982) condiciona la declaración del régimen jurídico especial para las zonas de Agricultura de Montaña, a aquellos territorios homogéneos que al menos se encuentren localizados en un $80 \%$ de su superficie en cotas superiores a los $1.000 \mathrm{~m}$. con la excepción đe las planicies cultivadas. Además deben contar con una pendiente superior al $20 \%$ ó comportar una diferencia entre las cotas extremas de su superficie agraria superior a los cuatrocientos metros (art. 2. " punto uno). Por otra parte se consideran "zonas equiparables" a espacios en los que no concurren las anteriores circunstancias. Son aquellos que quedan configurados por el Reglamento de la Ley (Decreto 2164 , de $31-10-84$ ), en cuanto su altitud se halle en un $80 \%$ 
En un repaso a la legislación europea se comprueba que la preocupación por el tema se adelanta en 5 ó 10 años, incluso la Ley de Agricultura de Montaña de Gran Bretaña data de 1946 (luego revisada en 1956) ${ }^{14}$.

Los objetivos de la Ley española, tal como concreta su artículo primero son para las Zonas de Agricultura de Montaña (ZAM), "su desarrollo social y económico especialmente en sus aspectos agrarios, manteniendo un nivel demográfico adecuado y atendiendo a la conservación y restauración del medio físico, como hábitat de sus poblaciones". La Ley, según SOSA WAGNER (1985), tiene un neto carácter de estímulo y fomento en contraposición a la Ley de Alta Montaña de Cataluña a la que se considera más organizativa territorial y funcionalmente. Sin embargo, el aspecto más contrastado de la Ley y su Reglamento (PEREZ GARCIA, 1985) es el intento de coordinación de las distintas administraciones y partidas sectoriales, instrumentadas mediante Programas -específicos para Zonas específicas-, de Ordenación y Promoción, de 4 ó más años de duración. Se propone un modelo de desarrollo endógeno, estimulado desde las instancias oficiales, comprometidas por aportaciones financieras mediante la firma de un Convenio. En éstos se regulan los incentivos económicos, se reparten las tareas en la Admón. Local, Autonómica y Estatal, y consecuentemente las responsabilidades de aplicación y posterior gestión articulada del Programa, en torno a un Comité de Coordinación de Zona tripartito. Este Comité, eleva en su caso al Gobierno, la Declaración de Zona y la aprobación de las ayudas contenidas en el Programa ${ }^{15}$.

por encima de los $600 \mathrm{~m}$. y la pendiente media sea superior al $10 \%$. Pero luego de estas características sobre el relieve, se tienen que cumplir otros condicionantes físicos, en base a limitaciones para las producciones agrarias: climáticas (según variables del Indice de L. Turc. Art. 2) y edáficas al menos el $80 \%$ de la superficie del territorio ha de estar incluida en la clasificacion de capacidades agrológicas de los suelos, propias de las zonas de montañas (según las clases diseñadas por las normas del Ministerio de Agricultura, Pesca y Alimentación). Las Comunidades Autónomas, en función a la configuración de su territorio y a la normativa propia derivada, podrán elevar o reducir en casos concretos los límites mínimos (art. 2, punto 2 de la Ley). Estos parámetros de marcada filosofía geográfica, no han tardado en suscitar polémica entre ciertos profesionales. En Extremadura, según GURRIA CASCON (1985), trás aplicarse los criterios topográficos de la Ley, se puede comprobar como quedarian incluidos dentro de las Zonas, unos 150 municipios con casi el $43 \%$ de la superficie regional. Resultados excesivos, pero lógicos por las peculiaridades del relieve extremeño. El autor anterior, concluye con la reflexión de que no son suficientes los criterios anteriores para determinar y delimitar espacios tan complejos; la alternativa debe realizarse bajo una óptica geográfica "que considera los distintos espacios como realidades únicas, como paisaje, que vienen definidos por complejas interrelaciones de factores físicos, económicos y humanos" (pag. 10).

14. Suiza contempla el tema desde 1974; la R.F.A. en 1975; Austria, Italia en 1976 y Francía en 1977. El desarrollo de la legislación gala es quizás el modelo seguido por la española. Los países integrados en la C.E.E. aprueban estas leyes en base a la Directriz Comunitaria, $75 / 268$ sobre Agricultuxa en las Regiones montañosas y otras zonas poco favorecidas, de 28 de Abril de 1975 . Ver Las Areas de Montaña en el Derecho Comparado, CEOTMA, Normativa 2, Madrid, 1981. $307 \mathrm{pp}$.

15. Estas son: fondo para transferencias de capital en favor de los agricultores que viven en la montaña; inversiones públicas en infraestructuras y servicios; ayudas especiales, dirigidas a explotaciones individuales y cooperativas; instalación de pequeñas y medianas empresas de carácter individual, familiar o cooperativo y ejecución de obras de imterés general por los montaneses a través de la formula de desarrollo comunitario. 
Esta ventaja de concentrar en un único cuerpo legislativo y en una misma competencia colegiada y paritaria, la gestión, tiene desde la perspectiva geo gráfica una apreciación importante, la delimitación de las ZAM. Es necesaric establecer un contenido planificador a los criterios de delimitación, ya quє por otra parte se detecta una insuficiencia clara de los criterios exclusivamen. te físicos, debiéndose contar con la posibilidad de integrar la planificación er. un área funcional, hecho aún no explicitado en la legislación. La Order. 10540 de 6 de Marzo de 1985, ha establecido la primera delimitación peri. metral de superficies susceptibles de ser declaradas ZAM. Para Andalucía s€ recogen 123 municipios (aproximadamente el $15 \%$ del total regional) ${ }^{16}$, los cuales han sido destacados en el mapa de superficies cultivadas. Estas áreas se corresponden a la Serranía de Ronda, Sierras de Cazorla y Segura, La Mágina, Hoyas de Guadix y Baza, Altiplanicies Orientales, Alpujarras (granadinas y almerienses), Tierra de Alhama y Sierra de Filabres. Fundamentalmente en el sector Oriental de la región. Hasta ahora no se han promulgado otros decretos sobre ZAM susceptibles de aprobación, pero en función del que hay, en cada Comunidad Autónoma, se pueden agregar o segregar, municipios contiguos o ya integrados, respectivamente, en esta primera delimitación (según la orden anteriormente citada). En estos momentos se está elaborando los proyectos para los diversos Programas Territoriales (Equipo VERDE ETUASA).

Sin entrar en una valoración general, por el incipiente proceso de desarrollo que la Ley lleva, se podrían realizar, por el contrario, tres precisiones territoriales en la delimitación de ZAM para Andalucía:

a) Los espacios delimitados (exceptuada la Serranía de Ronda) son excesivamente amplios para la gestión, si se interpreta que no habrá segmentación interior de las áreas orientales contiguas (aspecto solucionado por la "Predelimitación de ZAM", OM 6-3-85).

b) No se contemplan todavía otros territorios de montaña, que efectivamente no contienen los criterios físicos de delimitación, pero que sin embargo necesitarían instrumentos de fomento y desarrollo endógeno como éste: espacios de Sierra Morena, Axarquia, Subbético cordobés, etc.

c) Son áreas, tal como se comprueba en el mapa, donde la superficie cultivada es baja en general. Por tanto parece que los estímulos sobre actividades ganaderas, forestales y de otro tipo derivadas de éstas (Turismo Rural), deben ser las prioritarias.

16. No necesariamente, según se recoge del Reglamento de la Ley, tienen porque ser términos municipales completos. En la primera delimitación efectuada si lo son. 
Para concluir, pese a los defectos intrínsecos de carácter espacial en el diseño de la Ley (excesivo "determinismo" físico), es necesario concentrar las debidas esperanzas en medidas de este tipo. Es decir en formulas de fomento compreensivas de un abanico sectorial (agricultura y ganadería, forestal y las implicaciones en la industria, artesanía, cooperativas, turismo y electricidad, patrimonio artístico y medio natural), que intentan articular distintas figuras de acción territorial ${ }^{17}$. (DIAZ DEL OLMO y MOLINA, 1985) anteriormente promovidas (por ICONA, en los Planes Provinciales, en las Comarcas de Acción Especial o en las Zonas de Ordenación de Explotaciones, por ejemplo), en un mismo territorio. Será necesario aguardar a la puesta en práctica de los diferentes Programas aprobados (parece que la Serranía de Ronda, podría ser una de las primeras comarcas en aplicación, en Andalucía) y el seguimiento de las actuaciones, ya que el interés que puede suscitar en su extrapolación a otras zonas, en sus contenidos o con metodología similar, puede ser ilustrativo: se propone un modelo de desarrollo endógeno; se opera sobre áreas enclavadas; se pretende la especialización agraria de zonas que no comparten esencialmente la primacía agrícola del resto de la región. Todos argumentos deseables y en el marco de la planificación espacial.

17. Aunque lo que de ninguna manera estimamos aconsejable, sería la proyección de figuras territoriales paralelas a las prescritas por la Ley del Suelo, o por las diversas disposiciones que al respecto, se hiciesen a nivel de legislación territorial en la Comunidad Autónoma. 


\section{BIBLIOGRAFIA CITADA}

BER NAL, A.M.: "De nuevo la Reforma Agraria" en Noción Andaluza, 2-3. Granada, 1984, pp. 9-13.

BERNAL, A.M.: "Desarrollo económico y desequilibrio regional en Andalucia: la incidencia del sector agrícola". Revista de Estudios Andaluces, 2, Sevilla, 1984, pp. 15-30.

BOSQUE MAUREL, J.: "La España del Sur (Andalucía)" en Los países rurales españoles. A.G.E. Valladolid, 1980, pp. 191-205.

CANO GARCIA, G.: "Unidad y diversidad de la Geografía andaluza" en Revisto de Estudios Andaluces, 1. Sevilla, 1983, pp. 69-84.

CANO GARCIA, G.: "Comarcalización y Reforma Agraria en Andalucia" en Revista de Estudios Andaluces, 3. Sevilla, 1984, pp. 101-124.

CRUZ VILLALON, J.: "Transformaciones recientes en la agricultura andaluza" en Revista de Estudios Andaluces, 1. Sevilla, 1983, 69-84.

DIAZ OLMO, F. y MOLINA, F.: "Parques Naturales andaluces...". R.E.A., n. ${ }^{\circ} 4$ (1985), pp. 147-156.

DRAIN, M. y ROUX, B.: "El sector agrario en la economia andaluza" en Economia Andaluza. I.D.R., n. ${ }^{\circ}$ 16. Sevilla, 1978.

GARCIA MANRIQUE, E.: "Los nuevos paisajes agrarios. Los cultivos forzados" en Actas, Discursos, Ponencias y Mesas Redondas del VIII Coloquio de Geógrafos Españoles. Barcelona, 1983, pp. 99122.

GONZALEZ DELGADO, J.: Los cultivos agrícolas de Andalucía en cifras. Consejería de Agricultura y Pesca. Junta de Andalucía. Octubre, 1979, pp. 231.

GR UPO E.R.A.: Las Agriculturas andaluzas. Ministerio de Agricultura. Madrid, 1980, pp. 509.

GURRIA GASCON, J.C.: "La Ley de Agricultura de Montaña: la delimitación en la Comunidad Autónoma extremeña. Defectos y Alternativas" en 1. a Jornadas sobre problemas de los municipios de Montaña de la Cordillera Cantábrica. Pola de Lena. Mayo, 1985.

HERnANDEZ, J. y MARTINEZ, M.: Caracterización de las Comarcas Agrarias de Andalucía. Análisis de la Comarca Jaén-Martos. Cámara Oficial de Comercio e Industria de Jaén. 1980.

JORDA BORRELL, R.: Dinámica y distribución recientes de la población andaluza, I,D.R., Sevilla, 1985, pp. 80.

LOPEZ ONTIVEROS. Ag.: Las comarcas olivareras andaluzas. I.N.I.A., Madrid, 1982, pp. 197.

MARCHENA GOMEZ, M.: La distribución de la población en Andalucía (1960-81). Universidad de Sevilla y Diputación Provincial. Sevilla,1984, pp. 187.

MARQUEZ DOMINGUEZ. J.: La nueva agricultura onubense: protagonismo agrario del municipio de Moguer. Tesis de Licenciatura (Universidad de Sevilla), 1984. En prensa.

MARTINEZ SIERRA, F.: “Un intento de clasificación de las varias formas de la producción agraria presentes en Andalucía Oriental" en Revista de Estudios Regionales, n. ${ }^{\circ}$ 9. Málaga. Enero-Julio, 1982 .

OJEDA y OTROS: "La reforma agraria: repetida necesidad de la agricultura en el capitalismo", Revista de Estudios Andaluces, n. ${ }^{\circ}$ 3. Sevilla, 1984, pp. 85-100.

OJEDA, J.F.: Organización del territorio de Doñana y su entorno próximo (Almonte). Siglos XVIII. $X X$. Tesis Doctoral (Universidad de Sevilla), Junio, 1985. En prensa.

ORtega Alba, F.: El sur de Cordoba. Estudio de Geografía Agraria. Monte de Piedad y Caja de Ahorros. Córdoba, 1974, II tomos, $186 \mathrm{pp}$.

ORTEGA-VALCAR CEL, J.: "La economía de Montaña, una economía de equilibrio" en 1. "Jornadas sobre problemas..., Pola de Lena. Mayo, 1985.

PAYNO GALVARRIATO, J.A. (Director): Claves para el Desarrollo Económico de Andalucía, Junta de Andalucia-Banco Exterior de Espana. Sevilla, 1983, pp. 501. 
PEREZ GARCIA, R,: "La normativa española para el desarrollo de las Zonas de Agricultura de Montaña" en 1. “Jornadas sobre problemas..., Pola de Lena. Mayo, 1985.

ROMERO, J.J. y ZOIDO, F.: Colonización Agraria en Andalucía. I.D.R., n. 9 9. Sevilla, 1977.

SANCHEZ, P.: "Notas sobre la estructura de la agricultura andaluza" en Boletín Económico de la Junta de Andalucía, n. ${ }^{\circ} 0$. Sevilla, pp. 49-55.

SANTOS, N. y ZOIDO, F.: "Contribución al estudio de los regadíos serranos andaluces" en Actas del Coloquio Hispano-Francés sobre las areas de Montaña. Ministerio de Agricultura, Madrid, 1980. pp. 399-411.

SOSA WAGNER, F.: "Aspectos Jurídicos de las Areas de Montaña" en 1. a Jornadas sobre problemas ..., Pola de Lena. Mayo, 1985.

VARIOS: Criterios para un desarrollo agrario en Andalucía. Consejería de Agricultura, 1979, p. 257.

ZOIDO NARANJO, F.: "Los regadíos en la Baja Andalucía. Nota de síntesis e interpretación” en Los paises rurales españoles. A.G.E., Valladolid, 1980, pp. 191-205.

ZOIDO NARANJO, F.: "La nueva agricultura" en Historia de Andalucía. Ed. Planeta. Barcelona, 1982. Tomo VIII, pp. 333-363. 